\title{
Newsfronts
}

\section{Old Before Their Time}

At the normally youthful and vigorous age of 12 days, most PASG $^{-/-}$mice- if they've even survived this long-are shrunken, balding, and literally at death's door. Now, the scientists who developed this line are hoping these mice will offer new insights into the cellular and genetic processes involved in aging.

The end stage of cellular aging is known as senescence, when cellular division comes to a halt. Researchers have linked several conditions to entry into senescence, including excessive shortening of the telomeres (the protective caps at the ends of chromosomes), and have identified several genes for which alterations in regulation are associated with senescence.

One such gene is proliferation-associated SNF2-like gene (PASG), which is involved in the maintenance of DNA methylation, an important process by which gene expression is directly repressed at the chromosomal level. Decreases in methylation are often apparent in senescent cells, and researchers have linked PASG activity to cellular proliferation. To further explore the nature of this association, Robert Arceci and his colleagues at the Johns Hopkins University School of Medicine (Baltimore, MD) established a mouse line in which PASG functionality had been largely eliminated.

Because of embryonic lethality, PASG expression cannot be fully ablated, but by targeting three exons important to protein function, Arceci's group obtained a number of important phenotypic findings, presented in the 1 May issue of Genes and Development. PASG mutants showed a significant decrease in global DNA methylation, and only $40 \%$ of these mice lived more than a few days after birth. Surviving mice were considerably smaller than their wild-type littermates and showed phenotypic traits associated with aging, including balding, reduced fat deposition, cachexia, and unstable gait. Histology revealed defects in organ development, including incomplete alveolar expansion and decreased bone density, and none of the mice survived more than 24 days.

Cells from the PASG mutant mice displayed a number of morphological and genetic markers of senescence, and murine embryonic fibroblasts from these mice showed strongly diminished replicative capacity. These cells were also far more likely to accumulate changes in DNA content and chromosome number after only a few divisions.

Arceci's group describe their findings as the first direct evidence linking premature aging pathologies with disruption of PASGregulated DNA methylation, and they suggest that this work should offer the possibility for further studies regarding the relationship between epigenetic regulation, cellular senescence, and organismal aging, and may even illuminate mechanisms involved in the replicative dysfunction seen in cancerous cells.

\section{- Michael Eisenstein}

\section{Helping Rats Kick Coke}

New research in rats has identified a protein that seems to have an important role in the drug-seeking behaviors that manifest during withdrawal from cocaine.

Several studies have suggested that cocaine reduces signaling from Gi $\alpha$-coupled receptors in the brain, although the basis of this was unclear. It is certain that several proteins regulate the activity of $\mathrm{G} \alpha$ proteins, including the activator of $G$ protein signaling (AGS) family. One family member, AGS3, is relatively abundant in neurons and is capable of binding as many as four Gi $\alpha$ molecules, blocking their participation in cellular signaling.

Peter Kalivas and his colleagues at the Medical University of South Carolina (Charleston, SC) and Louisiana State University (New Orleans, LA) set out to determine whether AGS3 is involved in cocaine withdrawal-related behaviors, and present their findings in the 22 April issue of Neuron. They first treated rats with repeated doses of cocaine, then subjected them to withdrawal; after 3 weeks, a strong increase in AGS3 expression was apparent in the prefrontal cortex and the nucleus accumbens, brain regions known to mediate drug-seeking behaviors.

Kalivas' team generated a cell-permeable fusion peptide combining the HIV protein Tat with the Gio-binding $G$ protein regulator (GPR) domain from AGS3. Tat-GPR treatment strongly increased rats' response to cocaine, as measured by locomotor activity and stereotyped behavior. Rats that were subjected to withdrawal and then again exposed to cocaine also showed a strong increase in drug-seeking behavior if they were treated with Tat-GPR.

Treatment of rats with antisense oligonucleotides targeting AGS3 significantly affected their response to withdrawal. Again the researchers subjected the rats to cocaine withdrawal, followed by presentation with cocaine; animals that had received prior antisense perfusion showed a significant reduction in locomotor response to cocaine exposure as well as a strong decrease in cocaineseeking behavior in comparison with rats treated with a negative control oligonucleotide. In comparison, antisense treatment had no effect on food-seeking behaviors, indicating a drug-specific response.

The authors suggest that AGS3 may function importantly in mediating the behaviors observed following cocaine withdrawal, with the increased expression of AGS3 potentially altering dopaminergic signaling in the prefrontal cortex and triggering increased activation of brain regions associated with cocaine sensitivity and drug-seeking activity.

$-M . E$

\section{Spicy Treatment for Cystic Fibrosis}

Curcumin, the substance that gives curry its distinctive yellow color, may help reverse the effects of cystic fibrosis (CF), according to a study in mice.

$\mathrm{CF}$ is a common genetic disorder that affects about 30,000 American children and adults. In CF patients, defective chloride channels cause an accumulation of mucus in the lungs and other organs that results in increased risk of lung damage and infection, as well as impaired digestion and vitamin absorption. CF is usually fatal by age 30 .

The most common mutation associated with $\mathrm{CF}$ is $\triangle \mathrm{F} 508$, which causes the $\mathrm{CF}$ transmembrane conductance regulator (CFTR) protein to be retained in the endoplasmic reticulum (ER). Having previously shown that inhibitors of the ER calcium pump prompt the release of CFTR to the cell 